\title{
Expression of 3-hydroxyisobutyrate dehydrogenase among neural
} cells

\author{
Radovan Murín*, Andreas Schaer and Bernd Hamprecht
}

Address: Interfakultäres Institüt für Biochemie, Universität Tübingen, Hoppe-Seyler-Str. 4, Tübingen, Germany

* Corresponding author

from Annual Meeting of the Study Group Neurochemistry. International Conference of the Gesellschaft für Biochemie und Molekularbiologie 2006 (GBM 2006): Molecular pathways in health and disease of the nervous system

Witten, Germany. 28-30 September 2006

Published: 23 March 2007

BMC Neuroscience 2007, 8(Suppl I):P4 doi:10.1 186/I47I-2202-8-SI-P4

C 2007 Murín et al; licensee BioMed Central Ltd.

Branched-chain amino acids (BCAA) - isoleucine, leucine and valine - belong to a limited group of substances transported through the blood-brain barrier and serving as substrates for meeting brain energy demands. Previous studies have shown the ubiquitous expression of a branched-chain alpha-keto acid dehydrogenase among neural cells. This enzyme catalyzes the initial and rate-limiting step in the irreversible degradative pathway for the carbon skeleton of valine and the other two BCAA. Unlike the acyl-CoA derivates in the irreversible part of valine catabolism, 3-hydroxyisobutyrate can be released from cells by transport across the mitochondrial and plasma membranes. Therefore, to assess the ability of neural cells to make use of this valine derived carbon skeleton as a metabolic substrate, we have investigated the distribution of the enzyme processing this hydroxyl acid, 3-hydroxyisobutyrate dehydrogenase (3-HIBDH), in cultured neural cells. To achieve this, 3-HIBDH has been purified from rat liver to serve as antigen for the production of an antiserum. Affinity purified antibodies against 3-HIBDH specifically recognized the enzyme in liver and brain homogenates. Immunoblotting demonstrated the ubiquitous expression of 3-HIBDH among cultured macroglial (astroglia, oligodendroglia and ependymocytes) and neuronal cells. Furthermore, immunofluorescence doublelabeling of astroglial cells with antisera against 3-HIBDH and the mitochondrial marker pyruvate dehydrogenase localized 3-HIBDH to mitochondria. The expression of 3HIBDH in neural cells demonstrates their ability to engage in the catabolism of valine imported into the brain for generation of energy. 